\title{
Whole-body single-cell sequencing of the Platynereis larva reveals a subdivision into apical versus non-apical tissues
}

Kaia Achim ${ }^{1, \#}$, Nils Eling ${ }^{2,4, \#}$, Hernando Martinez Vergara ${ }^{1}$, Paola Yanina Bertucci ${ }^{1}$, Thibaut Brunet ${ }^{1}$, Paul Collier ${ }^{5}$, Vladimir Benes ${ }^{5}$, John C Marioni ${ }^{2,3,4}$ and Detlev Arendt ${ }^{1}$

${ }^{1}$ Developmental Biology Unit, European Molecular Biology Laboratory, D-69117

Heidelberg, Germany

${ }^{2}$ EMBL-European Bioinformatics Institute (EMBL-EBI), Wellcome Genome Campus, Cambridge CB10 1SD, UK

${ }^{3}$ Wellcome Trust Sanger Institute, Wellcome Genome Campus, Cambridge CB10 1SA, UK

${ }^{4}$ Cancer Research UK Cambridge Institute, University of Cambridge, Cambridge CB2 ORE, UK

${ }^{5}$ Genomics Core Facility, European Molecular Biology Laboratory, D-69117 Heidelberg, Germany

\# These authors contributed equally to this work.

Correspondence:

Detlev Arendt: arendt@embl.de

John Marioni: marioni@ebi.ac.uk 


\section{Abstract $_{(127 \text { words) }}$}

Animal bodies comprise a diverse array of tissues and cells. To characterise cellular identities across an entire body, we have compared the transcriptomes of single cells randomly picked from dissociated whole larvae of the marine annelid Platynereis dumerilii $^{1-4}$. We identify five transcriptionally distinct groups of differentiated cells that are spatially coherent, as revealed by spatial mapping ${ }^{5}$. Besides somatic musculature, ciliary bands and midgut, we find a group of cells located at the apical tip of the animal, comprising sensory-peptidergic neurons, and another group composed of non-apical neural and epidermal cells covering the rest of the body. These data establish a basic subdivision of the larval body surface into molecularly defined apical versus non-apical tissues, and support the evolutionary conservation of the apical nervous system as a distinct part of the bilaterian brain ${ }^{6}$.

\section{Introduction}

Animal bodies are composed of cells, which are organized into tissues and organs. Recently, a number of studies have applied single cell transcriptomics to assess cellular diversity within tissues, such as the vertebrate pancreas ${ }^{7}$, intestine ${ }^{8}$, or different brain parts such as cortex, basal ganglia or hypothalamus ${ }^{9-11}$. This approach allows the molecular characterisation of cell types within a given tissue, as well as an assessment of their heterogeneity. However, the sheer number of cells in conventional vertebrate or insect systems has so far hampered the comparison of cellular identities across tissue boundaries, or even across entire bodies. 
Other laboratories have focused on the molecular comparison of different tissues, referred to as tissue transcriptomics. This approach determines and compares the expression profiles of entire tissues via bulk RNAseq ${ }^{12,13,14,15}$, with the potential to compare across the entire body. Inherent to the approach however, tissue transcriptomics so far refer to the somewhat arbitrary, morphology-based dissection of the body into discernible units. This is relevant because, on the one hand, cell types of a given tissue or organ may be very divergent and on the other hand, similar cell types can populate different tissues. Therefore, while tissue transcriptomics allow in-depth and technically robust analyses and comparisons of larger cellular assemblies, these are somewhat arbitrarily defined and the method lacks the resolution to disentangle and molecularly define cell and tissue identities across entire bodies.

To advance on this, we establish the marine annelid Platynereis dumerilii, a molecular model species for development, evolution and neurobiology ${ }^{16,17}$, as experimental paradigm to explore how cellular identities compare and relate to each other across an entire animal body. We apply single-cell RNAseq to randomly sampled cells from the dissociated whole larvae at 48 hours post fertilization (hpf). The Platynereis larval body is especially suited for this task, as it already comprises various cell types and tissues. Besides larval body features (e.g., epidermal cells and ciliary bands) it contains early differentiating cells contributing to the later juvenile worm (musculature, brain and nerve cord $)^{18}$. It thus allows us to determine and compare cellular identities at an early stage of the life cycle when cell numbers are still relatively low.

Our whole-body analysis reveals that, at this stage, the larval annelid body comprises five groups of differentiated cells with distinctive expression profiles. In each of these groups, 
cells express sets of transcripts that together encode group-specific cellular modules representing cellular structures and functions that characterize these cells. While some of these groups and their defining modules, such as ciliary bands with motile cilia or larval musculature with striated myofibres, match larval morphology, others shed new light on the basic organization of the annelid body. We find a group of cells located at the apical tip of the animal that appears to be distinct from all other ectodermally-derived, nonapical cells of the body. While the apical cells specifically utilize photosensory and neurosecretory modules, the non-apical tissue instead shows specific expression of various extracellular matrix components - such as ligand-receptor pairs, cellular adhesion molecules, and conserved proteolytic enzymes. Comparative data indicate that this molecular subdivision into apical versus non-apical tissues is an important organisational feature of the body that appears to be conserved in animal evolution.

\section{Results}

\section{Single-cell RNA-seq identifies five groups of differentiated cells}

To explore cell type diversity on the whole organism level, we dissociated whole larvae of a marine annelid, P.dumerilii at $48 \mathrm{hpf}$ and randomly captured cells for single-cell RNA-sequencing (scRNA-seq) (Fig. 1). At this stage of development, the larva comprises of relatively few cells $(\sim 5000)$, but many differentiated cell types such as ciliated cells, neurons or myocytes are already present. The collected cells were optically inspected to exclude doublets, multiple cells or cell debris. Sequenced samples were further filtered computationally to remove low complexity transcriptomes, lowly- 
expressed genes and transcriptomic doublets (Extended Data Fig. 1, Methods). A total of 373 cells and 31300 transcripts passed filtering steps and were used for downstream analysis. To group the cells into distinct clusters we used a sparse clustering strategy, which identified seven groups of cells. We used the scran package to find group specific marker genes and discovered that in pairwise comparisons across all groups, two clusters were consistently highly similar to one other. Therefore, we merge these two closely related groups (Fig. 1, Extended Data Fig. 2, Methods).

To characterize the remaining six groups further, we identified differentially expressed genes (Methods). The largest group of cells was characterized by the specific expression of genes known to be active in developmental precursors, such as DNA replication (DNA topoisomerase, $R f c 3, R f c 5, M c m 3, M c m 4, M c m 7$ ), cell proliferation (Pcna, Md2l1, Apc7, Extended Data Fig. 2i), or chromatin remodeling (Nucleoplasmin, Bptf). We thus inferred that this group was comprised of undifferentiated cells.

Cells in the other five groups showed significantly lower expression of these markers (Extended Data Table 1, FDR $<0.1$, Methods) and we thus consider them differentiated cells. For each of these five groups, pairwise differential expression analysis revealed distinct sets of group-specific effector genes (i.e., differentially expressed genes that encode the particular structural and/or functional properties of cells) and group-specific transcription factors, many of which are known to act as terminal selectors in cell type differentiation $^{19,20}$ (Extended Data Table 2).

To validate the clustering of differentiated cells into the above groups, and to infer relationships between these groups, we used bootstrap resampling (Methods) to calculate a hierarchical tree (Extended Data Fig. 3). This tree confirmed each group's integrity, 
albeit with variable support. We then averaged the gene expression across cells in each group and used these values to calculate a tree comprising cell groups only (Fig. 3, upper panel). The resulting tree retrieved the group topology of the single cell tree with higher support values.

\section{Spatially coherent, molecularly defined larval tissues}

To characterize and localize the five distinct groups of differentiated cells with regard to larval morphology, we used a dual strategy. First, we investigated the spatial distribution in the larval body by mapping constituent cells into a cellular-resolution expression atlas ${ }^{5}$ (Fig. 2). To this end, we constructed a cellular resolution atlas of the 48 hpf larva (Extended Data Fig. 4), taking advantage of the existing Profiling by Signal Probability mapping (ProSPr) pipeline ${ }^{21}$ Using ProSPr, the majority $(95 \%)$ of cells could be mapped to distinct regions within the larva. Complementing this, we used wholemount in situ hybridization (WMISH) with probes targeted towards group-specific transcripts to refine the spatial mapping.

Following this strategy, cells from group 1 mapped to the most apical part of the larval ectoderm around the so-called apical organ (Fig. 2a-b). Consistent with this, the specific group 1 marker Phosphodiesterase-9 (Pde9) outlined a highly specific expression territory in the apical/dorsal episphere (Fig. 3a). With reference to the clear neural character of the cells in this group (see below), we refer to group 1 cells as 'apical nervous system'. Group 2 cells mapped into the yolk-rich macromeres where the first differentiating cells of the later midgut are located (Fig. 2c-d), as confirmed by the highly 
specific expression of Hnf4 in the cellularized portion of the macromeres (Fig. 3b). This group thus represents 'larval midgut'. Cells of group 3 mapped to the differentiating striated myocytes (Fig. 2e-f) and, in line with this, Striated muscle myosin heavy chain (St-mhc) was expressed in all cells belonging to 'striated musculature' (Fig. 3c). Next, group 4 cells mapped to the ciliary bands (Fig. 2g-h), which is composed of multiciliated cells with motile cilia. Expression of Radial spoke head protein homolog 4 (Rsph4) in the multiciliated cells of the ciliary bands of the apical organ, prototroch and paratrochs confirmed this mapping (Fig. 3d) ${ }^{18}$. Complementing this, group 5 cells covered much of the remaining larval surface, including non-apical territories in the larval trunk (Fig. 2i-j). The broad expression of the Metabotropic glutamate receptor 7 (Grm 7 ) in trunk and head clearly illustrates these 'non-apical surface cells' (Fig. 3e).

Notably, while the sorting of differentiated cells into ciliary bands, larval midgut, and striated musculature matched the subdivision of the larval body into well-known, morphologically distinct tissues, the assignment of ectodermally-derived cells into apical and non-apical populations did not correspond to an obvious morphological boundary. Interestingly, however, the topology of the (unrooted) hierarchical tree (Fig. 3, Extended Data Fig. 3) supported the general subdivision of the larval body into apical versus nonapical tissues - suggesting a closer relationship of apical nervous system and midgut on one hand, and of the non-apical tissues (non-apical surface, ciliary bands and striated musculature) on the other.

\section{The apical nervous system}


To further explore the different molecular nature of the five differentiated cell groups , we investigated their distinctive gene sets. Fig. 4 shows a collection of the most specific and informative regulatory and effector genes specific for each group.

Cells of the apical group consistently expressed the neuropeptide-cleaving Prohormone convertase $2(P h c 2)^{22}$ and many neuropeptides, such as the broadly expressed GNXQNpeptide or GGNamide, two lophotrochozoan neuropeptides ${ }^{23}$, indicating neurosecretory release from these cells. We also noted that cells in the apical group highly expressed non-calcium-binding members of the synaptotagmin family implicated in the generation and fusion of large dense core vesicles for neurosecretion, such as Synaptotagmin 17/B/K (Syt17), Syt $\alpha$ and Syt4 (Fig. 4a-b, Fig. 5, Extended Data Fig. 5) $)^{24-}$ 26. This is in line with previous reports of neurosecretory cells populating the dorsomedian larval brain ${ }^{22}$, and with ultrastructural observations, which show that cells in the dorsomedian Platynereis brain are rich in dense core vesicles while synapses are often sparse or absent ${ }^{27}$.

In addition, the bistable photopigment Peropsin ${ }^{28}$ and components of cGMP-based signal transduction such as $P d e 9$ and the CNG channel components $C n g b, C n g a-\alpha$ were broadly expressed in the cells of the apical group, indicative of light reception via vertebrate-type phototransduction $^{29}$ (Fig. 4a). Specific cells within this group co-expressed effector genes and transcription factors, such as c-opsin or c-opsin2 and Neuropeptide $Y$ (Npy) (representing brain ciliary photoreceptors ${ }^{29}$ ), or Vasotocin-neurophysin, Proenkephalin and c-opsin (representing photosensory-vasotocinergic cells ${ }^{22}$ ). Adding to this, some apical cells expressed the neural effector gene Neuroglobin, encoding a monomeric globin evolutionarily older than hemoglobin or myoglobin that reversibly binds oxygen 
and may represent an oxygen reservoir for highly metabolic neurons (Fig. $\mathbf{4 a})^{30}$. Other cells expressed Insulin-like peptide, Pigment dispersing factor (Pdf) or Allatostatin-C, and subsets of cells expressed the transcription factors Foxq2, Bsx and T-brain (Tbr I). In sum, we conclude that the apical ectodermal cells form part of the photosensoryneurosecretory dorsomedian brain ${ }^{4,22}$ and correspond to the 'apical nervous system' (ANS) as defined previously ${ }^{4,6,27,31}$.

\section{Midgut cells and striated musculature}

Cells allocated to the early differentiating midgut specifically expressed the midgutspecific transcription factor Hnf4 as well as Chymotrypsin-A, a digestion enzyme (Fig. 4b). In addition, this group was enriched in the expression of signal peptides such as CCWamide, PYpeptide, WLDpeptide, indicating that peptidergic neurosecretion is a characteristic feature of this group, similar to the apical nervous system (but using different neuropeptides).

The striated musculature cells were characterized by the relatively higher expression of St-mhc, Mrlc, Troponin-I and Troponin-T, which together are known to assemble striated myocyte contractile fibres ${ }^{32}$ (Fig. 4c), and by the heterogeneous expression of the myocyte-specific terminal selectors MyoD, Hand, Paraxis, Mox and Pitx ${ }^{33}$. We used WMISH and co-expression analysis using ProSPr to validate the expression of MyoD in a subset of muscles (Extended Data Fig. 5). Notably, we detected a low level of the striated myocyte markers in other cell groups (Fig. 4c). Given the exceptionally strong expression of these genes in the myocytes, and their up to 100 -fold lower expression in the other groups, it is yet unclear whether this finding represents biological or technical noise. 


\section{Non-apical surface cells and ciliary bands}

The cells of ectodermally-derived, non-apical surface and the ciliary band cells expressed different, partially overlapping gene sets indicative of mixed epithelial and neural characteristics (Fig. 4d). First, we identified a set of genes broadly co-expressed by nonapical surface ectoderm, ciliary bands and - consistently but at lower level - in striated musculature. These genes included $\operatorname{Lamin} A / C$, a nuclear fibrous protein forming the nuclear lamina on the interior of the nuclear envelope ${ }^{34}$ (Fig. 4d, Extended Data Fig. 5), and the related Neurofilament-70 (Nf70), a neural intermediate filament found in Lophotrochozoans (Fig. 4d). Interestingly, Lamins and cytoplasmic intermediate filaments evolved via duplication from an ancient, Lamin-like gene (with the intermediate filament precursor losing the nuclear localization sequence and the CaaX motif characteristic for Lamins, and giving rise to intermediate filaments and keratins several times independently ${ }^{35}$. In addition, this group comprised the conserved Extended Synaptotagmin 2 (Esyt2), with an apparent function in membrane lipid composition dynamics, extracellular signal transduction and cell adhesion ${ }^{36}$; and finally, P4ha2 encodes a component of prolyl 4-hydroxylase, a key enzyme in collagen synthesis (Fig. 4d).

Another gene set, shared between non-apical surface cells and ciliary bands, was enriched for extracellular matrix activities. For example, orthologs of Anoctamin, a transepithelial chloride channel ${ }^{37}$, Protocadherin-15 (Pcdh15), an atypical cadherin mediating structural integrity of ciliated sensory receptors ${ }^{38}$ (Extended Data Fig. 5), and Semaphorin 2 (Sema2), a secreted extracellular guidance molecule involved in axon pathfinding ${ }^{39}$ were 
co-expressed in these groups (Fig. 4d). Most noticeably, however, several genes encoded matrix-modifying proteases such as a serine protease Plasminogen related to vertebrate Plasmin $^{40}$ and Hepatocyte Growth Factor $(\mathrm{HGF})^{41}$ (Extended Data Table 2); metalloproteinases such as Matrix Metalloproteinase $(\mathrm{Mmp} 9)^{42}$ together with its specific substrate $C d 109^{43}$; and the cysteine proteinase Cathepsin $L^{44}$ (Fig. 6b). Regarding transcription factors, these groups shared the epidermis-specific transcription factor Grainyhead, known for its conserved role in maintaining the epidermal barrier ${ }^{45}$, and the ETS-homologous factor that likewise specifies epithelial cell type identities ${ }^{46}$ (Fig. 4d).

Likewise, the genes most specific for non-apical surface cells abundantly encoded proteins known to specifically interact in extracellular matrix and basal lamina (Fig. 4d, Fig. 6c) - many of which with reported roles in nervous system development and plasticity. For example, we identified orthologs of Neurotactin, which encodes a cell surface glycoprotein of the serine esterase superfamily ${ }^{47}$, and its specific binding partner Amalgam $^{48}$; both involved in axonal pathfinding in Drosophila ${ }^{47,49}$. We also found two orthologs of Neurotrypsin (Fig. 6a), a matrix-modifying serine protease, together with the extracellular proteoglycan grin $^{50}$, indicating that the Neurotrypsin-Agrin system is active in these cells ${ }^{51}$ (Fig. 4d). The Hspg2 gene, encoding a conserved proteoglycan related to Agrin, was also present. Besides extracellular matrix components, the nonapical surface cells also expressed $W n t$ ligands such as $W n t 4^{52}$, and the bHLH HES transcription factors Hes1, Hes2, Hes6 and Hes11. Homeodomain transcription factors with conserved roles in neuronal specification such as Uncx4, Tbx20 or Phox $2^{2,53,54}$ were expressed in subsets of cells in this group (Fig. 4d), indicating neuronal identities. 
Finally, we also identified a group of genes most specific for the ciliary bands: Rsph, Ift, Dynein and Kinesin effector genes, which are constituent parts of motile cilia (Fig. 4e, Extended Data Fig. 5, Extended Data Table 2) ${ }^{55,56}$. Notably, all of these genes were also expressed in other groups, albeit in a patchy manner and at lower level. While most of the ciliated cells mapped to the larval trunk, two of the ciliary band cells mapped to the head and co-expressed the transcription factors $R x$ and Tailless, which specify head regions and cell types in brain and eye in a broad spectrum of bilaterians ${ }^{57}$. This suggest that the ciliary band cells may split into distinct subgroups corresponding to the ciliary cells of the head vs trunk.

\section{Distributed nature of neural cells}

Interestingly, scRNAseq indicated that pan-neuronal differentiation genes such as Synaptotagmin1, Rab3, Munc13, Rims, Rimsbp and Complexin ${ }^{58}$ were expressed across all groups of differentiated cells (Fig. 7b). This is consistent with observations that neuronal features are not restricted to the nervous system ${ }^{58}$. For Platynereis, the ciliated cells of the larval prototroch are reported to show action potentials ${ }^{59}$ and therefore likely to also express neuronal markers; and the Platynereis differentiated midgut has recently been show to show autonomous contraction waves at juvenile stages ${ }^{60}$.

Upon examining the spatial expression pattern of pan-neuronal genes using WMISH we noted that their expression was highest in the apical nervous system and in the ventral portion of the non-apical, ectodermally-derived tissue (Fig. 7a), consistent with the known location and extent of the neuroectoderm at this stage ${ }^{2}$. With regard to the apical 
nervous system, a large fraction of its constituent cells indeed mapped in to the apical domain of Syt1 expression (Extended Data Fig. 6a). Substantiating this, we validated the co-expression of the pan-neuronal marker genes and apical cell group markers by

\section{WMISH (Fig. 7a).}

With regard to the non-apical surface cells, spatial mapping showed that some of those cells also mapped into the Syt1 expression domain on the ventral body side (Extended Data Fig. 6b), supporting their neuronal character. ProSPr analysis likewise confirmed an overlap of non-apical surface markers with the pan-neuronal marker $\operatorname{Rab3}$ (Fig. 7a). Adding to this, we validated the expression of the non-apical group marker Neurotrypsin in the ventral neuroectoderm by WMISH (Fig. 6a). Together, these data indicate that, at least at this larval stage, 'neurons' did not fall into one homogenous group (in contrast to striated myocytes or cells with motile cilia).

\section{Discussion}

For this study we analyzed the transcriptomes of 373 cells randomly collected from dissociated, entire larvae of the marine annelid Platynereis dumerilii. Among these we identified 123 differentiated cells, chosen for further analysis and comparison. Previous studies had captured cells from specific body parts and tissues only (such as the mouse forebrain $^{9,10}$ ); our data is unique as it allows single-cell level comparison of differentiated cells across the entire body. We were able to sort all differentiated cells into five major groups, based on similarities and differences in gene expression profiles. Spatial mapping into the $48 \mathrm{hpf}$ ProSPr cellular-resolution expression atlas, in combination with 
wholemount in situ hybridisation of marker genes, identified these groups as apical nervous system, larval midgut, ciliary bands, non-apical surface cells, and striated musculature.

What is the nature of these groups? Interestingly, within each of the five groups, there existed considerable heterogeneity in the expression of transcription factors and effector genes (as evident from Fig. 4), suggesting the presence of several cell types within each group. For example, cells of the apical nervous system differ with regard to opsin expression, neuropeptide content, and signaling components - indicating that each, or almost each of the cells might represent a separate type. Therefore, at the current level of analysis the five groups of differentiated cells rather appear to represent some kind of molecularly defined larval tissues - uniting cells with similar molecular properties shared beyond the cell type level. We believe that the number of sequenced cells would have to be increased by at least one order of magnitude to resolve individual cell types within these groups.

Comparing the cell groups further, we noted an unexpected divide between ectodermallyderived cells in the most apical part of the larva (apical nervous system), versus the nonapical surface cells and ciliary bands. Cells of the apical nervous system shared the expression of two major sets of modules, enabling the synthesis and secretion of neuropeptides, and mediating the detection of ambient light for the circadian control of body physiology. Most of the specifically expressed effector genes detected for these modules, encoding for example prohormone convertase, specific neuropeptides, components of the phototransductory cascade, and ciliary opsins, have previously been implicated in apical nervous system function ${ }^{4,22}$. 
In contrast, the genes characteristic for the non-apical surface cells revealed mixed epithelial and neural properties. One broadly expressed set of genes - found in non-apical surface, ciliary band and striated muscle cells - encoded proteins relating to basic properties of the surface epidermis - such as stabilizing intermediate filaments (related to vertebrate keratins), and an enzyme mediating collagen synthesis into the extracellular matrix. Another set of genes - shared between non-apical surface and ciliary band cells, but absent from myocytes - included matrix-modifying proteinases, many of which implicated in tissue remodeling, axonogenesis and synapse formation in insects and vertebrates $^{40}$. And yet another set - identified as most specific for the non-apical surface cells proper - included genes encoding neural properties, such as Neurotactin/Amalgam, Neurotrimin, and Semaphorin involved in axon guidance and Neurotrypsin/Agrin with reported roles in synaptogenesis ${ }^{51,50}$. This would indicate that the non-apical surface cells utilize specific molecular toolkits to modify extracellular matrix, guide outgrowing axons, and establish synapses - that are not employed in the apical nervous system.

The specific and broad expression of the nicotinic acetylcholine receptor alpha-9 in nonapical surface cells is also noteworthy. In vertebrates, this unconventional receptor plays restricted roles in the mechanosensory hair cells, where it is involved in the reception of efferent signals ${ }^{61}$, and in epidermal keratinocytes, where it triggers epithelialization via local, non-neuronal acetylcholine cytotransmission ${ }^{62}$.

What is the significance of the fundamental divide between apical and non-apical cells in the Platynereis larval surface ectoderm? While some of the genes that are differentially expressed between apical and non-apical cell groups relate to functions carried out by fully differentiated cells (e.g., neurosecretion, phototransduction, cholinergic 
cytotransmission), others indicate earlier stages of differentiation (e.g., proteolytic cleavage for extracellular matrix modification, and axonal guidance). Thus, and given that our analysis only covers one single stage of larval development, we cannot rule out that some of the differences between apical and non-apical cells are due to differences in developmental timing; more extensive studies including additional stages will be required to solve this issue. However, preliminary WMISH of 'apical' and 'non-apical' marker genes already indicates that expression differences between apical and non-apical tissues are also observed at earlier developmental and later life-cycle stages (Extended Data Fig. 7). Developmental timing is therefore unlikely to fully account for the apical/nonapical differences.

Another possible explanation for the difference between apical and non-apical cells is evolutionary divergence. This notion assumes that an ancient subdivision of the animal body into functionally and structurally different parts occurred early in animal evolution. These segregating parts would have started to differ in epithelial properties, extracellular matrix remodeling capacities and cell-cell communication strategy. To validate and substantiate this notion, similar datasets from both, closely and remotely related species will be required; they should show similar overall molecular tissue subdivisions if these were evolutionarily conserved.

As a starting point, and in line with the second option, the restricted activity of apical nervous system marker genes in the vertebrate forebrain indicates evolutionary conservation of the apical nervous system and of its specific properties ${ }^{6}$. For example, in vertebrates the neurosecretory-specific Syt4 is specifically active in the neuroendocrine hypothalamus, where it regulates release of the neuropeptide Oxytocin ${ }^{63}$. Syt17, initially 
isolated from the vasopressin-secreting supraoptic and paraventricular nuclei, also shows strong expression in the hypothalamus ${ }^{64}$, as does Neuroglobin ${ }^{65-67}$. The T-box transcription factor T-brain is specifically expressed in the developing para- and periventricular and supraoptic hypothalamic nuclei ${ }^{68}$; and finally, expression of ciliary opsins and other genes of the ciliary photosensory cascade in the vertebrate retina and pineal indicates evolutionary conservation of apical nervous system components ${ }^{29}$. These similarities add to previously reported conserved features shared between vertebrate hypothalamus and annelid apical nervous system, involving specifying transcription factors $^{69}$, a neurosecretory centre releasing conserved neuropeptides and hormones ${ }^{22,27}$, and non-visual ciliary photoreceptors ${ }^{29}$ active in melatonin synthesis and the control of circadian behavior ${ }^{4}$. Our transcriptomics data thus support the evolutionary conservation of the apical nervous system as a distinct part of the bilaterian brain, specialized on the perception of ambient light for the control of body physiology via the release of neuropeptides and hormones. They are furthermore consistent with the recent 'chimeric brain' model, which stipulates that the apical nervous system evolved as an independent center of neuronal condensation in the course of bilaterian brain evolution ${ }^{6}$.

\section{METHODS}

\section{Cell capture and sequencing}

Platynereis larvae were cultured, the cells were dissociated, and single-cell cDNA synthesis was performed using the Fluidigm C1 Single-Cell Auto Prep System, and the sequencing libraries were prepared as previously described ${ }^{5}$. We used Fluidigm IFCs for mRNA-seq with capture sites for 5-10 $\mu \mathrm{m}$ and 10-17 $\mu \mathrm{m}$ to collect the cells, allowing the 
capture of a full range of cell sizes in $48 \mathrm{hf}$ stage of Platynereis. In total, 9 IFC-s, each representing an independent collection of cells derived from a different batch of animals, were processed for this study. The information about the samples distribution on chips, the chip sizes, cell number per sample and the pooling of samples on sequencing lanes described in Extended Data Table 3. 100 bp paired-end sequences were generated using the Illumina HiSeq2000 platform. For one library (CN62), 75 bp paired-end sequences were generated using the Illumina NextSeq500 platform. Libraries were sequenced to an average depth of 2.1 million reads.

\section{Data availability}

The raw sequence data for the cells analysed in this study are available from ArrayExpress, accession numbers E-MTAB-2865 (see Extended Data Table 5 for matching the cell ID-s between the E-MTAB-2865 and this study) and E-MTAB-XXXX (the accession number will be updated as the dataset is released). The $\mathrm{R}$ code used for analysis is available at: https://github.com/MarioniLab/Platynereis2017

\section{Read alignment}

FastQC $^{70}$ was used for the quality trimming of the raw sequencing reads using default settings. For read mapping, the quality-filtered sequencing reads were mapped, using bowtie2, against a Platynereis reference transcriptome combined from the two previously published assemblies $^{5,23}$. Briefly, the transcriptome assemblies were concatenated and contigs showing more than 94\% identities were removed using CD-HIT ${ }^{71}$, leaving 44977 transcripts. In thus generated reference, each gene should be represented by one 
transcript, reducing the problem of multiple mapping of the reads. Expression counts for each transcript were obtained using HTSeq $^{72}$ while only considering uniquely mapped reads.

\section{Gene annotation}

To annotate genes of the two transcriptome assemblies ${ }^{5,23}$, reciprocal BLAST comparison of genes sequences against the Uniref90 protein database (Arendt assembly) or against Swissprot (Jékely assembly) was performed. For each transcript, the BLAST hit with highest E-value was selected for annotation.

\section{Quality control}

Low-quality cells were removed from the dataset based on the following filtering criteria. Visual inspection of capture sites on the IFC revealed empty wells, wells with multiple cells or debris captured. Only wells with positive cell capture were further processed for sequencing. For downstream analysis, libraries marked as single cells were chosen. Cells with less than 1000 reads mapping to ERCC spike-ins, less than 100,000 mapped transcriptomic reads or less than $60 \%$ of mapped reads being allocated to the transcriptome were removed. Additionally, cells that express unusually small number of genes $(<2000)$ were removed. Prior to normalization, genes with more than $1,000,000$ reads or less than 10 reads across all cells were removed.

\section{Normalization}


The BASiCS package ${ }^{73}$ was used to normalize read counts by incorporating ERCC spikeins for technical noise estimation. Specific ERCC spike-ins were removed if not detected in the dataset. Posterior estimates for model parameters were computed by Markov chain Monte Carlo (MCMC) simulation with 40,000 iterations.

The BASiCS_DenoisedCounts function was utilized to compute normalized read counts. Running the MCMC independently on the different batches confirmed similar small levels of technical noise in the data (Extended Data Fig. 1h),

\section{Spatial mapping}

Spatial mapping of the sequenced single-cell transcriptomes onto the Platynereis reference atlas was performed as described previously ${ }^{5}$. The Platynereis reference atlas is provided as Extended Data Table 4 and the spatial mapping results for all the sequenced cells are provided in Extended Data Table 5. We defined functional regions within the embryo based on spatial expression patterns of known marker genes. The region in which Phc2 is expressed comprises the apical nervous system. The muscle region of the animal is defined by the expression of St-mhc. Foxj expression defines the ciliated cells and Hnf4 expression the midgut region. The trunk ectoderm can be described by Uncx 4 expression. To assign mapped cells to particular regions, we identified the voxel cluster with highest mapping confidence for each cell. For visualizing the mapping of individual cells, the centroids of all voxel clusters were plotted. To compute the overlap of mapped cells to spatial gene expression patterns, we focused on the cluster with highest mapping confidence for each cell. If more than $50 \%$ of these voxels also show a particular gene expression (e.g. Syt1), we consider the cell as falling into this gene region. 


\section{Detecting highly variable genes}

The BASiCS_DetectHVG function in BASiCS allows the detection of highly variable genes by incorporating spike-ins to estimate expected technical variability. Testing was done after MCMC simulation with a variance threshold of 0.98 and an evidence threshold of 0.7 .

\section{Clustering}

To detect clusters of cells based on the expression of highly variable genes, we used a sparse K-means clustering approach taken from the sparcl package in $\mathrm{R}^{74,75}$. We clustered the data using $K=2, \ldots, 10$ and chose $K=7$ since only small subpopulations emerged at $\mathrm{K}=8, \ldots, 10$. An elbow plot showing the averaged within-cluster sum of squares is non-informative for the choice of K. AWSS $=\left(\sum_{k} \sum_{i} \sum_{j}\left(x_{i j k}-\mu_{i k}\right)^{2}\right) / m$, where $x_{i j k}$ is the $\log _{10^{-}}$transformed transcript count of gene $\mathrm{i}$ in cell $\mathrm{j}$ and cluster $\mathrm{k}, \mu_{i}$ the $\log _{10^{-}}$ transformed mean expression of gene $\mathrm{i}$ in cluster $\mathrm{k}$ and $m$ the number of clusters. The parameters were tuned with $2<$ wbounds $<100$ using 15 permutations. Genes with weights $>0.03$ define the cluster-characteristic features of the data.

\section{Removing cell doublets}

Possible cell doublets were removed after clustering and marker gene detection. The percentage of group specific marker gene expression (\# marker genes expressed/\# all 
genes) was calculated for each cell. Cells with unusually high expression of markers nonspecific for their group were removed from the dataset.

\section{Tree building}

To investigate the transcriptional similarity between cell types, we hierarchically clustered cell types before and after averaging gene expression across all cells within each group. The averaging strategy increases stability of the hierarchical clustering algorithm by averaging out dropout events in single cell data. Distances were calculated based on the Spearman dissimilarity: $\sqrt{\frac{1}{2}\left(1-r_{S}\right)^{76}}$. To compute bootstrapped p-values, we used the pvclust package in $\mathrm{R}^{77}$. Hierarchical clustering was performed on the $\log _{10^{-}}$ transformed expression counts before and after averaging within each group using the spearman dissimilarity as distance, complete linkage clustering and 1000 bootstrap iterations to evaluate cluster stability.

\section{Differential expression}

To identify cell group specific enriched marker genes, we used two approaches. First, we used scde package ${ }^{78}$ to compare each of the identified cell group against all the remaining samples in order to identify novel group specific genes from the unannotated portion of the Platynereis transcriptome. These genes were then annotated, added to the reference, and the clustering was repeated. Second, differential expression analysis was performed using the findMarkers function from the scran package ${ }^{62}$. This approach uses limma ${ }^{79}$ on the $\log _{2}$-transformed, normalized counts. Group specific marker genes were defined as 
differentially expressed genes with a FDR $<0.1$ and $\log _{2} \mathrm{FC}>0$ for all pairwise comparisons. Initially, differential expression was tested between all 7 detected groups. Group 6 and group 7 consistently grouped together when hierarchical clustering (default options from the hclust package) was performed on the $\log _{2}$ fold changes in expression between one group and all others (Extended Data Fig. 2). We therefore merged group 6 and group 7 and tested differential expression between the now largest group and all other groups (Extended Data Table 1). In the next step, we tested differential expression pairwise between all differentiated cell groups (Extended Data Table 2).

\section{In situ hybridization}

For in situ hybridization (ISH), we collected 48 hpf or $72 \mathrm{hpf}$ Platynereis larvae. Animals were fixed overnight in $4 \%$ PFA/1.5xPBS and ISH was performed as described previously $^{80}$

\section{Cloning of Platynereis genes and ISH probe synthesis}

For the cloning of $P$. dumerilii cDNA sequences, wild-type Platynereis RNA was isolated by Trizol/phenol/chloroform extraction method, reverse transcribed using SuperScriptIII reverse transcriptase (Life Technologies, cat. \# 18080044), and amplified by PCR using TaKaRa ExTaq DNA polymerase (Clontech, cat. \# RR001A) and respective gene specific primers. The resulting PCR fragments were cloned into pCRIITOPO vector (Life Technologies, cat \# K4610-20). The cloned gene sequences and the gene specific primer sequences are available upon request. 
For the synthesis of ISH probes, cDNA plasmids were linearized and antisense RNA probes were transcribed using SP6 or T7 RNA polymerase (Roche, cat. \#11487671001 and 10881775001, respectively) and DIG RNA-labeling mix (Roche, cat. \#11277073910) or Fluorescein RNA labeling mix (Roche, cat. \# 11685619910).

\section{Microscopy and image processing}

For imaging of WMISH samples for ProSPr, samples were mounted in 97\% 2,2'thiodiethanol (Sigma, cat. \# 166782) and imaged on a Leica TCS SP8 confocal microscope, using a combination of fluorescence and reflection microscopy ${ }^{81}$. The colocalization analyses and image post-processing was performed using Fiji ${ }^{82}$ software. The colorimetric WMISH samples were imaged on Zeiss AXIO Imager M1 microscope. The figure panels were compiled using Adobe Illustrator and Adobe Photoshop software. 


\section{REFERENCES}

1 Tomer, R., Denes, A., Tessmar-Raible, K. \& Arendt, D. Cellular resolution expression profiling reveals common origin of annelid mushroom bodies and vertebrate pallium. Cell 142, 800-809 (2010).

2 Denes, A. S. et al. Molecular architecture of annelid nerve cord supports common origin of nervous system centralization in bilateria. Cell 129, 277288, doi:10.1016/j.cell.2007.02.040 (2007).

3 Randel, N. et al. Neuronal connectome of a sensory-motor circuit for visual navigation. Elife 3, doi:10.7554/eLife.02730 (2014).

4 Tosches, M. A., Bucher, D., Vopalensky, P. \& Arendt, D. Melatonin signaling controls circadian swimming behavior in marine zooplankton. Cell 159, 4657, doi:10.1016/j.cell.2014.07.042 (2014).

5 Achim, K. et al. High-throughput spatial mapping of single-cell RNA-seq data to tissue of origin. Nat Biotechnol 33, 503-509, doi:10.1038/nbt.3209 (2015).

6 Tosches, M. A. \& Arendt, D. The bilaterian forebrain: an evolutionary chimaera. Curr Opin Neurobiol 23, 1080-1089, doi:10.1016/j.conb.2013.09.005 (2013).

7 Muraro, M. J. et al. A Single-Cell Transcriptome Atlas of the Human Pancreas. Cell Syst 3, 385-394 e383, doi:10.1016/j.cels.2016.09.002 (2016).

8 Grun, D. et al. Single-cell messenger RNA sequencing reveals rare intestinal cell types. Nature 525, 251-255, doi:10.1038/nature14966 (2015).

9 Zeisel, A. et al. Brain structure. Cell types in the mouse cortex and hippocampus revealed by single-cell RNA-seq. Science 347, 1138-1142, doi:10.1126/science.aaa1934 (2015).

10 Tasic, B. et al. Adult mouse cortical cell taxonomy revealed by single cell transcriptomics. Nat Neurosci 19, 335-346, doi:10.1038/nn.4216 (2016).

11 Romanov, R. A. et al. Molecular interrogation of hypothalamic organization reveals distinct dopamine neuronal subtypes. Nat Neurosci 20, 176-188, doi:10.1038/nn.4462 (2017).

12 Ramskold, D., Wang, E. T., Burge, C. B. \& Sandberg, R. An abundance of ubiquitously expressed genes revealed by tissue transcriptome sequence data. PLoS Comput Biol 5, e1000598, doi:10.1371/journal.pcbi.1000598 (2009).

13 Breschi, A. et al. Gene-specific patterns of expression variation across organs and species. Genome Biol 17, 151, doi:10.1186/s13059-016-1008-y (2016).

14 Henrichsen, C. N. et al. Segmental copy number variation shapes tissue transcriptomes. Nat Genet 41, 424-429, doi:10.1038/ng.345 (2009).

15 Brawand, D. et al. The evolution of gene expression levels in mammalian organs. Nature 478, 343-348, doi:10.1038/nature10532 (2011).

16 Williams, E. A. \& Jekely, G. Towards a systems-level understanding of development in the marine annelid Platynereis dumerilii. Curr Opin Genet Dev 39, 175-181, doi:10.1016/j.gde.2016.07.005 (2016).

17 Fischer, A. \& Dorresteijn, A. The polychaete Platynereis dumerilii (Annelida): a laboratory animal with spiralian cleavage, lifelong segment proliferation and a mixed benthic/pelagic life cycle. Bioessays 26, 314-325, doi:10.1002/bies.10409 (2004). 
18 Fischer, A. H., Henrich, T. \& Arendt, D. The normal development of Platynereis dumerilii (Nereididae, Annelida). Front Zool 7, 31, doi:10.1186/1742-9994-7-31 (2010).

19 Hobert, O. Regulation of terminal differentiation programs in the nervous system. Annu Rev Cell Dev Biol 27, 681-696, doi:10.1146/annurev-cellbio092910-154226 (2011).

20 Arendt, D. et al. The origin and evolution of cell types. Nat Rev Genet 17, 744757, doi:10.1038/nrg.2016.127 (2016).

21 Vergara, H. M. et al. A whole-organism cellular gene expression atlas reveals conserved cell type clusters in the ventral nerve cord of Platynereis dumerilii. Proc Natl Acad Sci $U$ S A 114, 5878-5885, doi:10.1073/pnas.1610602114 (2016).

22 Tessmar-Raible, K. et al. Conserved sensory-neurosecretory cell types in annelid and fish forebrain: insights into hypothalamus evolution. Cell 129, 1389-1400, doi:10.1016/j.cell.2007.04.041 (2007).

23 Conzelmann, M. et al. The neuropeptide complement of the marine annelid Platynereis dumerilii. BMC Genomics 14, 906, doi:10.1186/1471-2164-14906 (2013).

24 Moghadam, P. K. \& Jackson, M. B. The functional significance of synaptotagmin diversity in neuroendocrine secretion. Front Endocrinol (Lausanne) 4, 124, doi:10.3389/fendo.2013.00124 (2013).

25 Gustavsson, N., Wu, B. \& Han, W. Calcium sensing in exocytosis. Adv Exp Med Biol 740, 731-757, doi:10.1007/978-94-007-2888-2_32 (2012).

26 Park, D., Li, P., Dani, A. \& Taghert, P. H. Peptidergic cell-specific synaptotagmins in Drosophila: localization to dense-core granules and regulation by the bHLH protein DIMMED. J Neurosci 34, 13195-13207, doi:10.1523/JNEUROSCI.2075-14.2014 (2014).

27 Williams, E. A. et al. Synaptic and peptidergic connectome of a neurosecretory centre in the annelid brain. bioRxiv (2017).

28 Nagata, T., Koyanagi, M., Tsukamoto, H. \& Terakita, A. Identification and characterization of a protostome homologue of peropsin from a jumping spider. J Comp Physiol A Neuroethol Sens Neural Behav Physiol 196, 51-59, doi:10.1007/s00359-009-0493-9 (2010).

29 Arendt, D., Tessmar-Raible, K., Snyman, H., Dorresteijn, A. W. \& Wittbrodt, J. Ciliary Photoreceptors with a Vertebrate-Type Opsin in an Invertebrate Brain. Science 306, 869-871 (2004).

30 Burmester, T., Weich, B., Reinhardt, S. \& Hankeln, T. A vertebrate globin expressed in the brain. Nature 407, 520-523, doi:10.1038/35035093 (2000).

31 Arendt, D., Tosches, M. A. \& Marlow, H. From nerve net to nerve ring, nerve cord and brain--evolution of the nervous system. Nat Rev Neurosci 17, 61-72, doi:10.1038/nrn.2015.15 (2016).

32 Schiaffino, S. \& Reggiani, C. Fiber types in mammalian skeletal muscles. Physiol Rev 91, 1447-1531, doi:10.1152/physrev.00031.2010 (2011).

33 Dobi, K. C., Schulman, V. K. \& Baylies, M. K. Specification of the somatic musculature in Drosophila. Wiley Interdiscip Rev Dev Biol 4, 357-375, doi:10.1002/wdev.182 (2015). 
34 Kim, Y., McDole, K. \& Zheng, Y. The function of lamins in the context of tissue building and maintenance. Nucleus 3, 256-262, doi:10.4161/nucl.20392 (2012).

35 Peter, A. \& Stick, R. Evolutionary aspects in intermediate filament proteins. Curr Opin Cell Biol 32, 48-55, doi:10.1016/j.ceb.2014.12.009 (2015).

36 Herdman, C. \& Moss, T. Extended-Synaptotagmins (E-Syts); the extended story. Pharmacol Res 107, 48-56, doi:10.1016/j.phrs.2016.01.034 (2016).

37 Pedemonte, N. \& Galietta, L. J. Structure and function of TMEM16 proteins (anoctamins). Physiol Rev 94, 419-459, doi:10.1152/physrev.00039.2011 (2014).

38 Seiler, C. et al. Duplicated genes with split functions: independent roles of protocadherin15 orthologues in zebrafish hearing and vision. Development 132, 615-623, doi:10.1242/dev.01591 (2005).

39 Bates, K. E. \& Whitington, P. M. Semaphorin 2a secreted by oenocytes signals through plexin B and plexin A to guide sensory axons in the Drosophila embryo. Dev Biol 302, 522-535, doi:10.1016/j.ydbio.2006.10.015 (2007).

40 Sonderegger, P. \& Matsumoto-Miyai, K. Activity-controlled proteolytic cleavage at the synapse. Trends Neurosci 37, 413-423, doi:10.1016/j.tins.2014.05.007 (2014).

41 Tyndall, S. J. \& Walikonis, R. S. The receptor tyrosine kinase Met and its ligand hepatocyte growth factor are clustered at excitatory synapses and can enhance clustering of synaptic proteins. Cell Cycle 5, 1560-1568, doi:10.4161/cc.5.14.2918 (2006).

42 Michaluk, P. et al. Influence of matrix metalloproteinase MMP-9 on dendritic spine morphology. J Cell Sci 124, 3369-3380, doi:10.1242/jcs.090852 (2011).

43 Vadon-Le Goff, S., Hulmes, D. J. \& Moali, C. BMP-1/tolloid-like proteinases synchronize matrix assembly with growth factor activation to promote morphogenesis and tissue remodeling. Matrix Biol 44-46, 14-23, doi:10.1016/j.matbio.2015.02.006 (2015).

44 Felbor, U. et al. Neuronal loss and brain atrophy in mice lacking cathepsins B and L. Proc Natl Acad Sci U S A 99, 7883-7888, doi:10.1073/pnas.112632299 (2002).

45 Boglev, Y. et al. The unique and cooperative roles of the Grainy head-like transcription factors in epidermal development reflect unexpected target gene specificity. Dev Biol 349, 512-522, doi:10.1016/j.ydbio.2010.11.011 (2011).

46 Feldman, R. J., Sementchenko, V. I. \& Watson, D. K. The epithelial-specific Ets factors occupy a unique position in defining epithelial proliferation, differentiation and carcinogenesis. Anticancer Res 23, 2125-2131 (2003).

47 Speicher, S. et al. Neurotactin functions in concert with other identified CAMs in growth cone guidance in Drosophila. Neuron 20, 221-233 (1998).

48 Zeev-Ben-Mordehai, T. et al. Amalgam, an axon guidance Drosophila adhesion protein belonging to the immunoglobulin superfamily: overexpression, purification and biophysical characterization. Protein Expr Purif 63, 147-157, doi:10.1016/j.pep.2008.09.019 (2009). 
49 Fremion, F. et al. Amalgam is a ligand for the transmembrane receptor neurotactin and is required for neurotactin-mediated cell adhesion and axon fasciculation in Drosophila. EMBO $J$ 19, 4463-4472, doi:10.1093/emboj/19.17.4463 (2000).

50 Stephan, A. et al. Neurotrypsin cleaves agrin locally at the synapse. FASEB J 22, 1861-1873, doi:10.1096/fj.07-100008 (2008).

51 Matsumoto-Miyai, K. et al. Coincident pre- and postsynaptic activation induces dendritic filopodia via neurotrypsin-dependent agrin cleavage. Cell 136, 1161-1171, doi:10.1016/j.cell.2009.02.034 (2009).

52 Pruitt, M. M., Letcher, E. J., Chou, H. C., Bastin, B. R. \& Schneider, S. Q. Expression of the wht gene complement in a spiral-cleaving embryo and trochophore larva. Int J Dev Biol 58, 563-573, doi:10.1387/ijdb.140084ss (2014).

53 Nomaksteinsky, M. et al. Ancient origin of somatic and visceral neurons. BMC biology 11, 53, doi:10.1186/1741-7007-11-53 (2013).

54 Rabe, T. I. et al. The transcription factor Uncx4.1 acts in a short window of midbrain dopaminergic neuron differentiation. Neural Dev 7, 39, doi:10.1186/1749-8104-7-39 (2012).

55 Thomas, J. et al. Transcriptional control of genes involved in ciliogenesis: a first step in making cilia. Biol Cell 102, 499-513, doi:10.1042/BC20100035 (2010).

56 Brooks, E. R. \& Wallingford, J. B. Multiciliated cells. Curr Biol 24, R973-982, doi:10.1016/j.cub.2014.08.047 (2014).

57 Joly, J. S., Recher, G., Brombin, A., Ngo, K. \& Hartenstein, V. A Conserved Developmental Mechanism Builds Complex Visual Systems in Insects and Vertebrates. Curr Biol 26, R1001-R1009, doi:10.1016/j.cub.2016.08.017 (2016).

58 Stefanakis, N., Carrera, I. \& Hobert, O. Regulatory Logic of Pan-Neuronal Gene Expression in C. elegans. Neuron 87, 733-750, doi:10.1016/j.neuron.2015.07.031 (2015).

59 Jekely, G. et al. Mechanism of phototaxis in marine zooplankton. Nature 456, 395-399, doi:10.1038/nature07590 (2008).

60 Brunet, T. et al. The evolutionary origin of bilaterian smooth and striated myocytes. Elife 5, doi:10.7554/eLife.19607 (2016).

61 Murthy, V., Taranda, J., Elgoyhen, A. B. \& Vetter, D. E. Activity of nAChRs containing alpha9 subunits modulates synapse stabilization via bidirectional signaling programs. Dev Neurobiol 69, 931-949, doi:10.1002/dneu.20753 (2009).

62 Chernyavsky, A. I., Arredondo, J., Vetter, D. E. \& Grando, S. A. Central role of alpha9 acetylcholine receptor in coordinating keratinocyte adhesion and motility at the initiation of epithelialization. Exp Cell Res 313, 3542-3555, doi:10.1016/j.yexcr.2007.07.011 (2007).

63 Zhang, G. et al. Neuropeptide exocytosis involving synaptotagmin-4 and oxytocin in hypothalamic programming of body weight and energy balance. Neuron 69, 523-535, doi:10.1016/j.neuron.2010.12.036 (2011). 
64 Lee, M. Y., Choi, S. H., Shin, S. L., Chin, H. \& Kwon, O. J. Distribution of B/K protein in rat brain. Cell Tissue Res 303, 47-56 (2001).

65 Fabrizius, A. et al. Critical re-evaluation of neuroglobin expression reveals conserved patterns among mammals. Neuroscience, doi:10.1016/j.neuroscience.2016.07.042 (2016).

66 Hundahl, C. A. et al. Circadian behaviour in neuroglobin deficient mice. PLoS One 7, e34462, doi:10.1371/journal.pone.0034462 (2012).

67 Hundahl, C. A. et al. Neuroglobin in the rat brain: localization. Neuroendocrinology 88, 173-182, doi:10.1159/000129698 (2008).

68 Dominguez, L., Gonzalez, A. \& Moreno, N. Patterns of hypothalamic regionalization in amphibians and reptiles: common traits revealed by a genoarchitectonic approach. Front Neuroanat 9, 3, doi:10.3389/fnana.2015.00003 (2015).

69 Marlow, H. et al. Larval body patterning and apical organs are conserved in animal evolution. BMC biology 12, 7, doi:10.1186/1741-7007-12-7 (2014).

70 Andrews, S. FastQC: a quality control tool for high throughput sequence data. Available online at: http://www.bioinformatics.babraham.ac.uk/projects/fastqc/ (2010).

71 Li, W. \& Godzik, A. Cd-hit: a fast program for clustering and comparing large sets of protein or nucleotide sequences. Bioinformatics 22, 1658-1659, doi:10.1093/bioinformatics/btl158 (2006).

72 Anders, S., Pyl, P. T. \& Huber, W. HTSeq--a Python framework to work with high-throughput sequencing data. Bioinformatics 31, 166-169, doi:10.1093/bioinformatics/btu638 (2015).

73 Vallejos, C. A., Marioni, J. C. \& Richardson, S. BASiCS: Bayesian Analysis of Single-Cell Sequencing Data. PLoS Comput Biol 11, e1004333, doi:10.1371/journal.pcbi.1004333 (2015).

74 Witten, D. M. \& Tibshirani, R. A framework for feature selection in clustering. J Am Stat Assoc 105, 713-726, doi:10.1198/jasa.2010.tm09415 (2010).

75 R Core Team. R: A language and environment for statistical computing., $<$ http://www.R-project.org/> (2014).

76 Stijn van Dongen, A. J. E. Metric distances derived from cosine similarity and Pearson and Spearman correlations. arXiv:1208.3145 (2012).

77 Ryota Suzuki, H. S. Pvclust: an R package for assessing the uncertainty in hierarchical clustering. Bioinformatics 22, 1540-1542 (2006).

78 Kharchenko, P. V., Silberstein, L. \& Scadden, D. T. Bayesian approach to single-cell differential expression analysis. Nat Methods 11, 740-742, doi:10.1038/nmeth.2967 (2014).

79 Ritchie, M. E. et al. limma powers differential expression analyses for RNAsequencing and microarray studies. Nucleic Acids Res 43, e47, doi:10.1093/nar/gkv007 (2015).

80 Tessmar-Raible, K., Steinmetz, P. R., Snyman, H., Hassel, M. \& Arendt, D. Fluorescent two-color whole mount in situ hybridization in Platynereis dumerilii (Polychaeta, Annelida), an emerging marine molecular model for evolution and development. Biotechniques 39, 460, 462, 464 (2005). 
81 Jekely, G. \& Arendt, D. Cellular resolution expression profiling using confocal detection of NBT/BCIP precipitate by reflection microscopy. Biotechniques 42, 751-755 (2007).

82 Schindelin, J. et al. Fiji: an open-source platform for biological-image analysis. Nat Methods 9, 676-682, doi:10.1038/nmeth.2019 (2012).

83 Fukuda, M. Molecular cloning and characterization of human, rat, and mouse synaptotagmin XV. Biochem Biophys Res Commun 306, 64-71 (2003).

84 Adolfsen, B., Saraswati, S., Yoshihara, M. \& Littleton, J. T. Synaptotagmins are trafficked to distinct subcellular domains including the postsynaptic compartment. J Cell Biol 166, 249-260, doi:10.1083/jcb.200312054 (2004). 


\section{Acknowledgements}

We thank Catalina Vallejos for comments on the implementation of BASiCS and all members of the Marioni and Arendt labs for feedback on the project and manuscript.

\section{Author contributions}

KA and NE planned and conducted experiments, interpreted the data, designed the study and wrote the manuscript. HMV, PB and PC conducted experiments. TB interpreted data. VB, JCM and DA funded the study. JCM and DA designed the study and wrote the manuscript.

\section{Author information}

The authors declare no competing interest. 


\section{Figures and Figure legends}

\section{Figure 1}
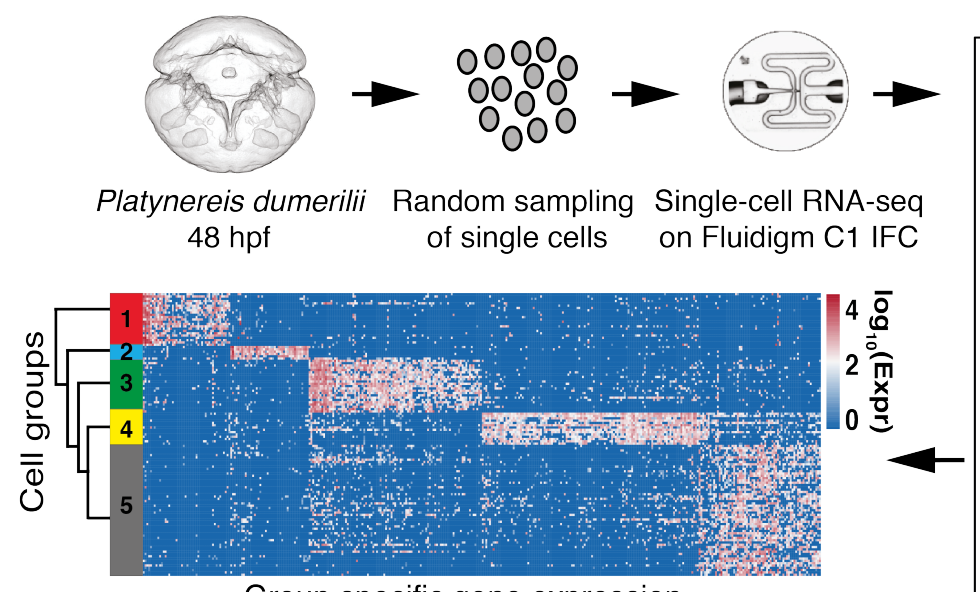

Group specific gene expression

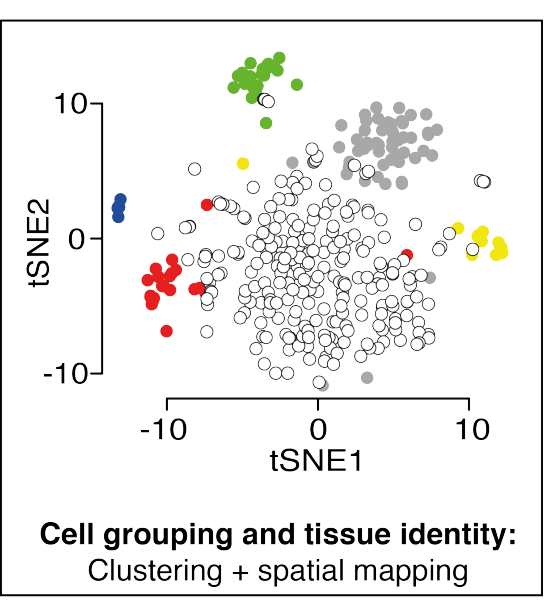

\section{Figure 1. Single cell transcriptomics of Platynereis 48 hpf larvae.}

Cells of the 48 hpf larvae were dissociated and randomly selected for single cell RNAsequencing using the Fluidigm C1 Single-cell AutoPrep system. Combining sparse clustering with spatial positioning of single cells allows the identification of robust cell groups within the data. The clustering approach enables identification of genes that characterise each cell type. Finally, we used hierarchical clustering to investigate the similarity between the identified cell clusters. 


\section{Figure 2}

a

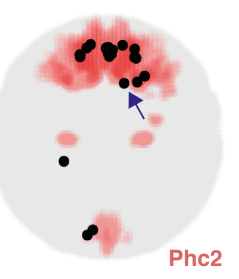

c

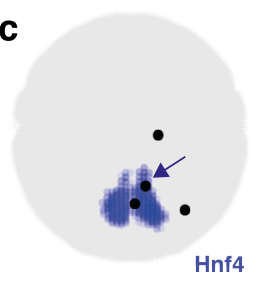

e

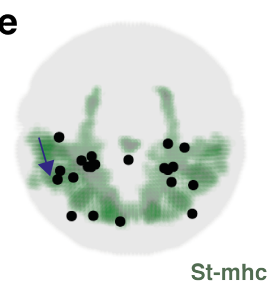

g

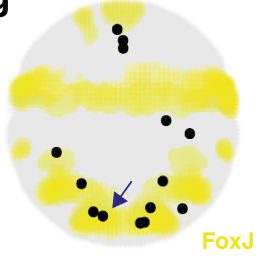

i

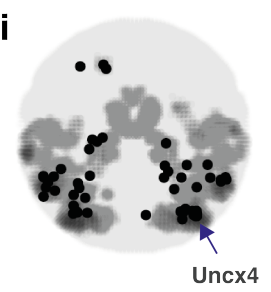

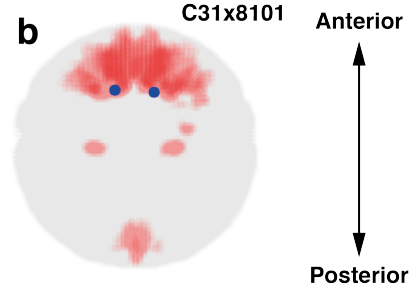

d

C5x2301L

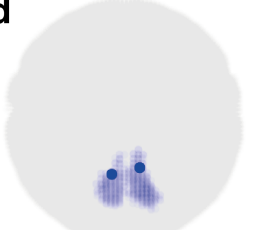

f

C11x0501

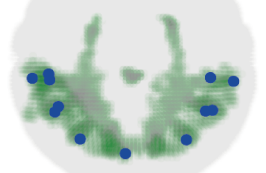

h

C22×0201L
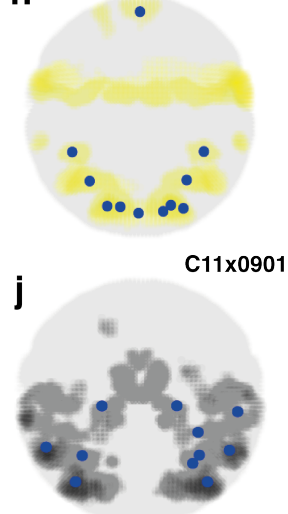

Figure 2. Spatial mapping of single cells to tissues characterized by specific marker gene expression.

a-j, Left panels: Centroids of the voxel clusters to which cells were mapped with highest confidence are shown as black dots. Tissue specific marker gene expression is visualized as colored regions within the embryo. Left panels: Mapping results for all cells belonging to the respective group. Right panels: For each cell group, we show an example of the 
mapping result for one individual cell (indicated by an arrow on the left panel). a, Cells of the apical ectoderm group ( $\mathrm{n}=23$ cells) map to the $P h c 2$ expressing region in the embryo. b, Mapping result for the cell C31x8101. $\mathbf{c}$, Midgut cells ( $\mathrm{n}=4$ cells) map to the Hnf4 expressing region. d, Mapping result for the cell C5x2301L. e, Striated musculature cells $(\mathrm{n}=23$ cells) map to the St-mhc expressing region. f, Mapping result for the cell C11x0501. g, Cells of the ciliary bands $(\mathrm{n}=14$ cells) map to the Foxj expressing region. h, Mapping result for the cell C22x0201L. i, Cells of the non-apical ectodermal group (n = 55 cells) map to the Uncx4 expression domain. $\mathbf{j}$, Mapping result for the cell C11x0901. 
Figure 3
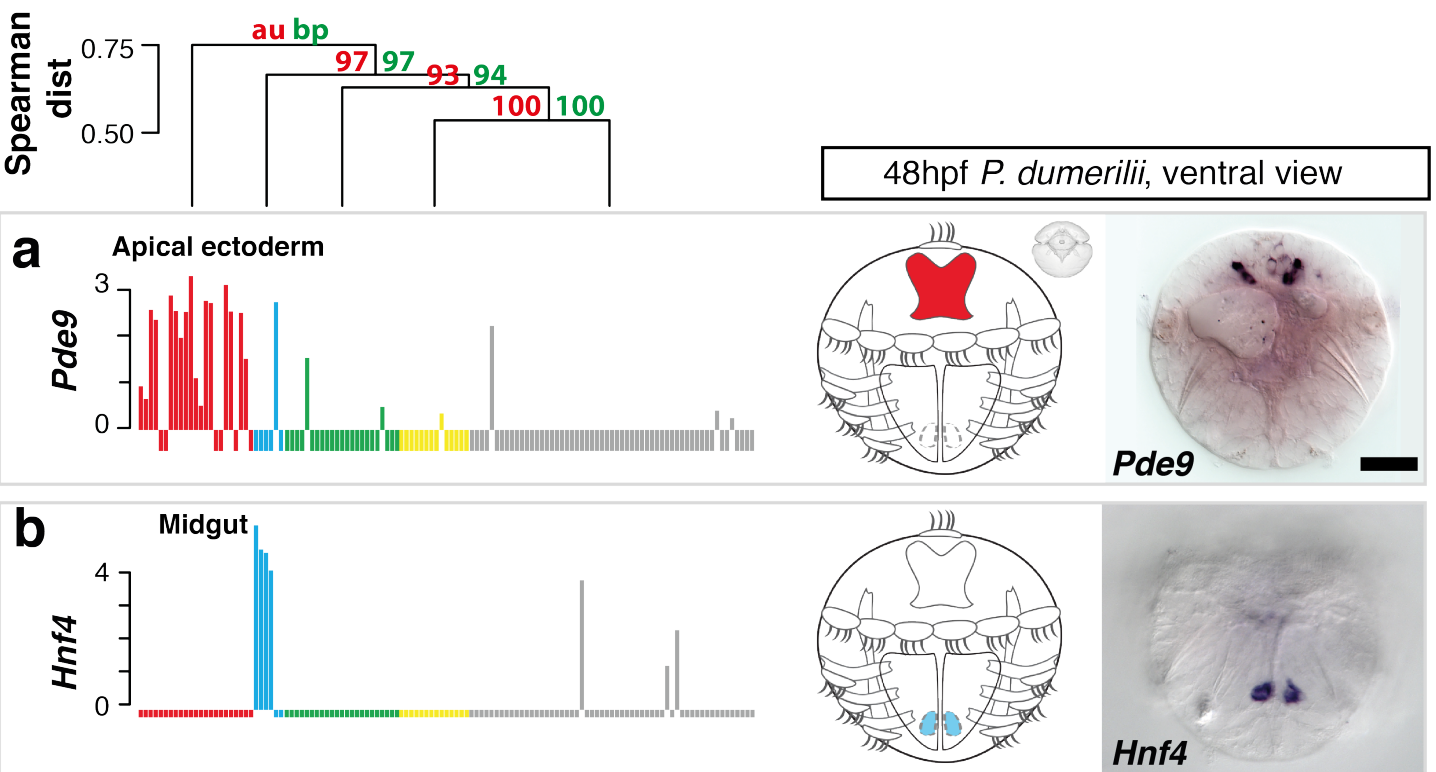

Pde9

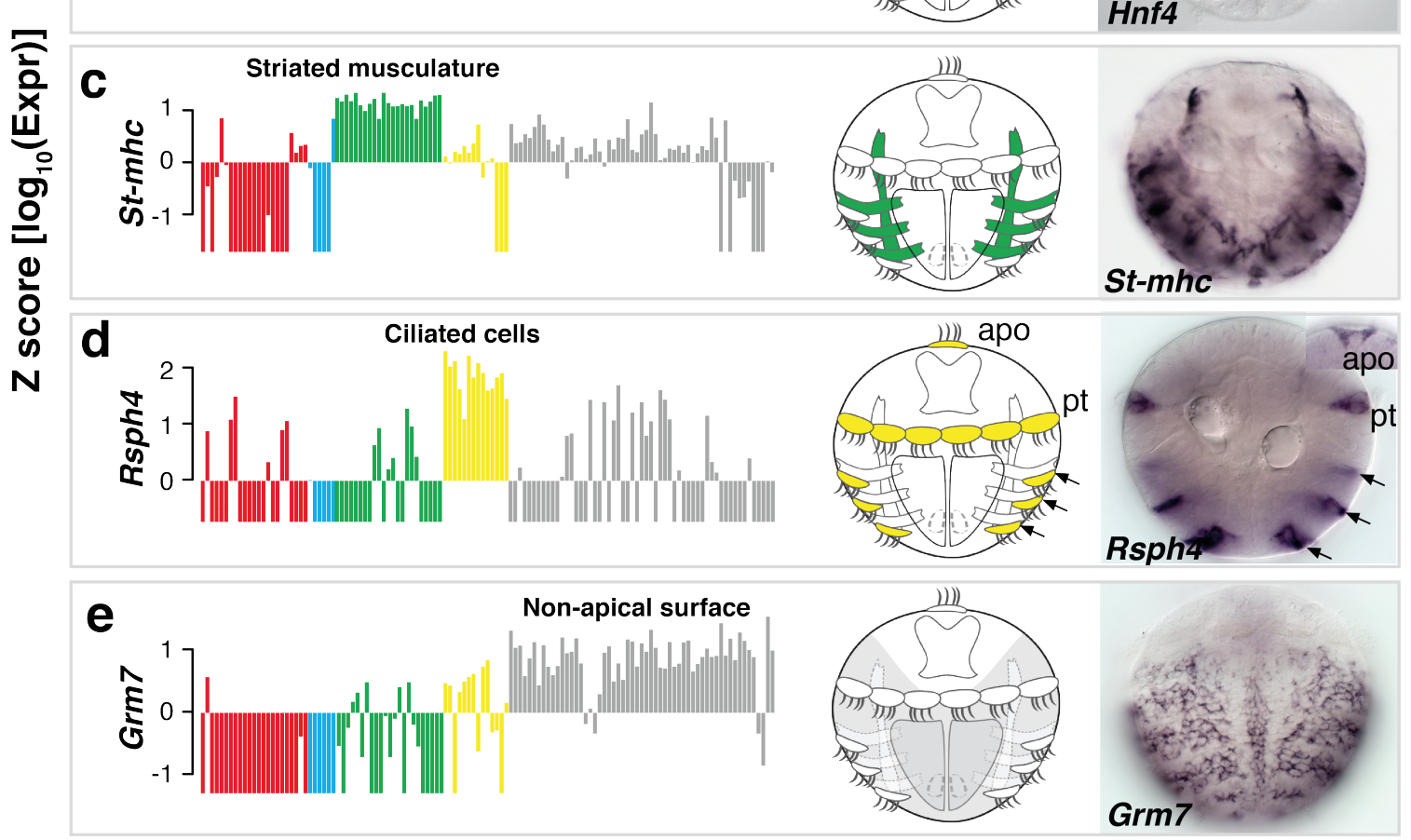

Figure 3. Identification and validation of tissue-specific genes.

On top, Distance tree showing the hierarchical relationships between the differentiated cell groups. a-e, For each identified cell group, the expression of a group-specific marker gene is shown in a bar plot, the respective tissue is shown schematically in the ventral view of Platynereis larva, and visualized by WMISH with respective probes: a, Pde9 
expression in the apical ectoderm (red); b, Hnf4 expression in the midgut (cyan); c, St$m h c$ expression in striated muscle (green); d, Rsph4 expression in ciliated cells (yellow); e, Grm7 expression characterizes the non-apical surface cells (gray). Note that Pde9, Hnf4, Rsph4 and Grm7 are novel markers for the respective cell groups. Each ISH pattern was replicated in at least 6 animals. Scale bar, $50 \mu \mathrm{m}$. Apo, apical organ; pt, prototroch, Z factor $=\frac{x_{i j}-\mu_{i}}{\sigma_{i}}, x_{i j}$ : Expression of gene $\mathrm{i}$ in cell $\mathrm{j}, \mu_{i}$ : Mean expression of gene $\mathrm{i}, \sigma_{i}$ : Standard deviation of gene i. 


\section{Figure 4}

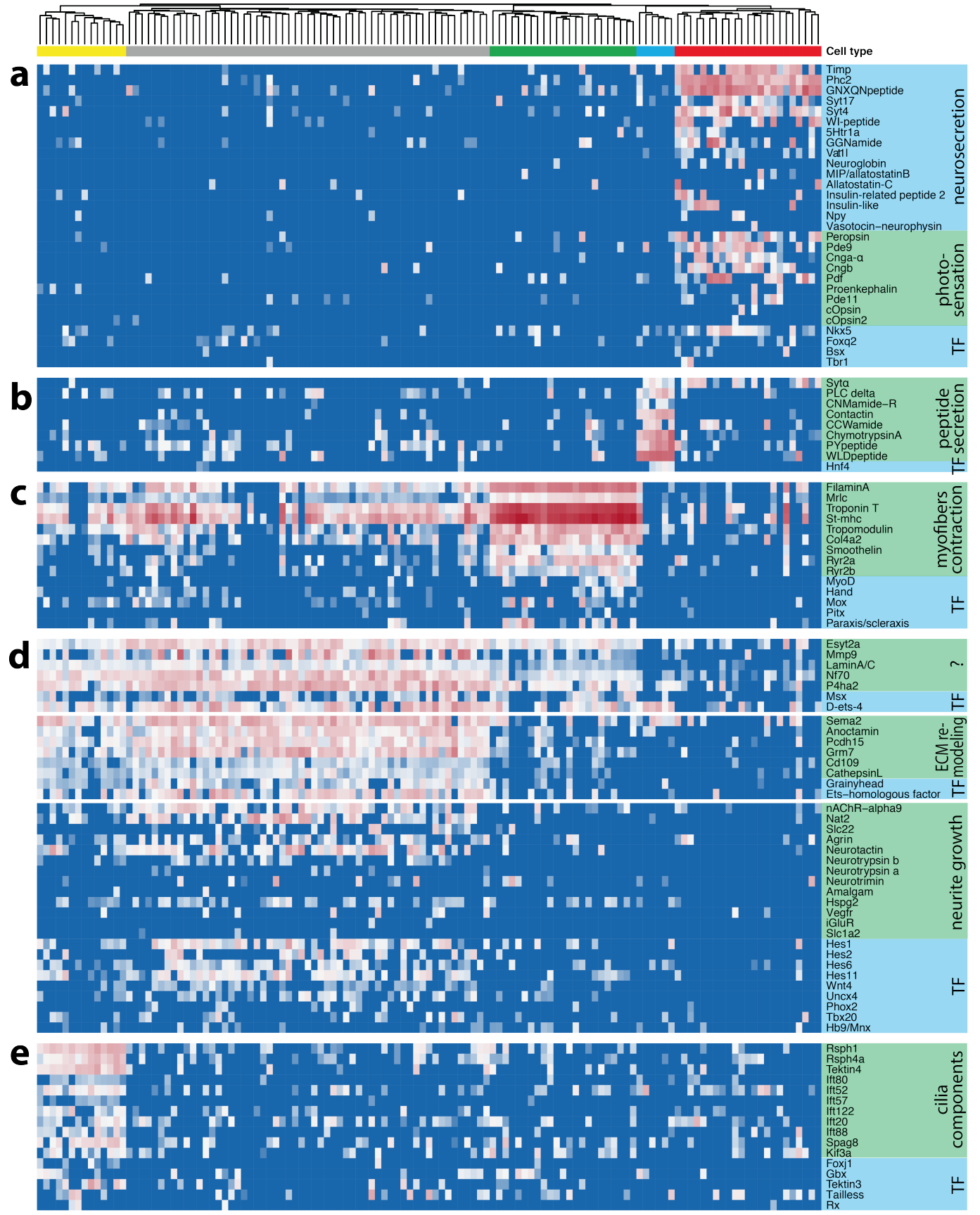

Figure 4. Tissue specific marker genes reflect cellular functions. For each of the differentiated tissues, we show a heatmap of tissue specific gene expression: a, expression profile of ANS-specific genes; b, expression profile of midgut genes; $\mathbf{c}$, expression profile of ciliated cell-specific genes; d, expression profile of non-apical 
bioRxiv preprint doi: https://doi.org/10.1101/167742; this version posted July 24,2017 . The copyright holder for this preprint (which was not certified by peer review) is the author/funder. All rights reserved. No reuse allowed without permission.

surface-specific genes; and e, expression profile of myocyte-specific genes. Functionally related groups of genes are highlighted. 


\section{Figure 5}

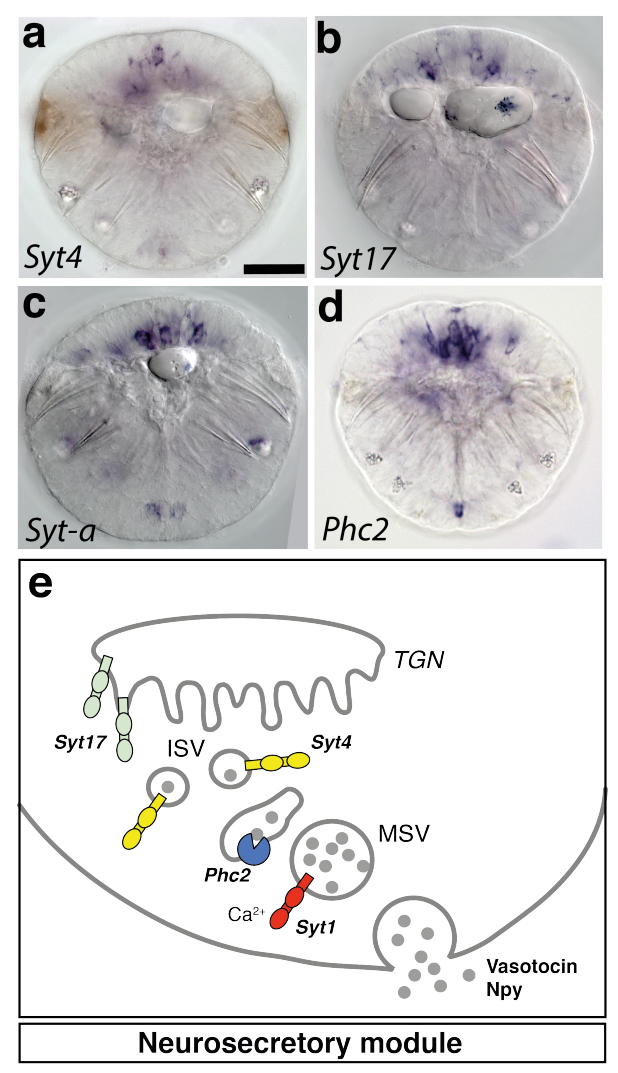

Figure 5. Neurosecretory functional module is specific to ANS cells. a-d, WMISH analysis of Syt4, Syt17, Syt $\alpha$ and Phc2 expression in Platynereis larvae at 48hpf. Ventral view. Scale bar, $50 \mu \mathrm{m}$ e, Schematic of the neurosecretion cellular module. Syt17 contains an N-terminal cysteine cluster mediating membrane association with the transGolgi network (TGN) ${ }^{83}$. Syt4 is involved in the maturation of secretory vesicles ${ }^{63}$. Syt $\alpha$ functions in vesicle trafficking of specific subclasses of neuropeptides and/or neuromodulators $^{84}$. TGN, trans-golgi network; ISV, immature secretory vesicle, MSV, mature secretory vesicle. 


\section{Figure 6}

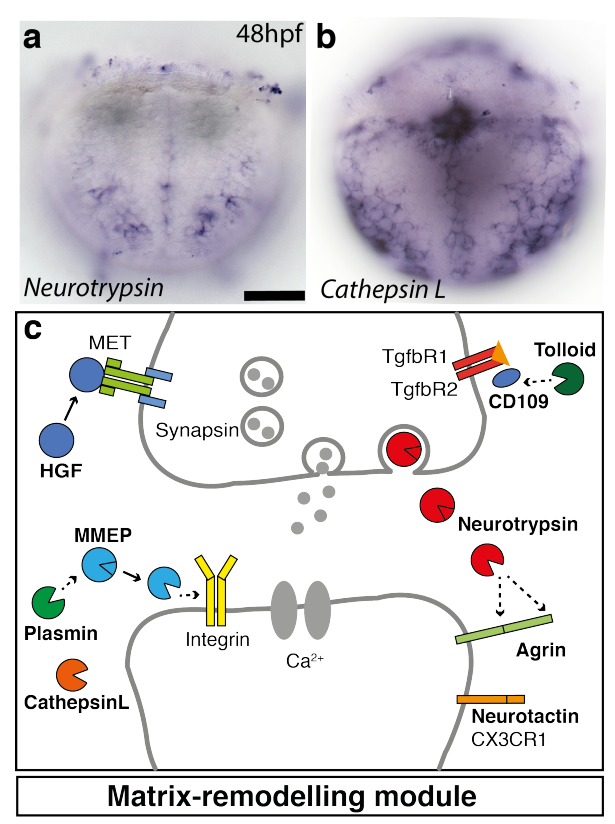

Figure 6. Extracellular matrix remodeling module is specific to non-apical surface cells. a-b, WMISH analysis of Neurotrypsin and Cathepsin L expression in Platynereis larvae at $48 \mathrm{hpf}$. Ventral view focused at the surface. Scale bar, $50 \mu \mathrm{m}$ c, Schematic of the synapse formation cellular module. Neurotrypsin cleaves agrin locally at the synaptic cleft, triggering the formation of new synapses ${ }^{40}$. HGF and its receptor MET enhance clustering of synaptic proteins at excitatory synapses ${ }^{41}$. Plasmin cleaves selected synaptic target proteins such as NMDA receptor and matrix metalloproteinases ${ }^{40}$. Several members of the family of matrix-metalloproteinases such as MMP-9 have been in synapse formation and remodeling ${ }^{42}$. Tolloid-like (Tll) and its substrate CD109 have been implicated in the control of $\mathrm{TGFb}$ signaling and extracellular matrix synthesis ${ }^{43}$. Cathepsin $L$ is likewise implicated in extracellular matrix remodeling and neuronal survival $^{44}$. The genes specifically expressed in non-apical ectodermal cells are marked in bold. 


\section{Figure 7}

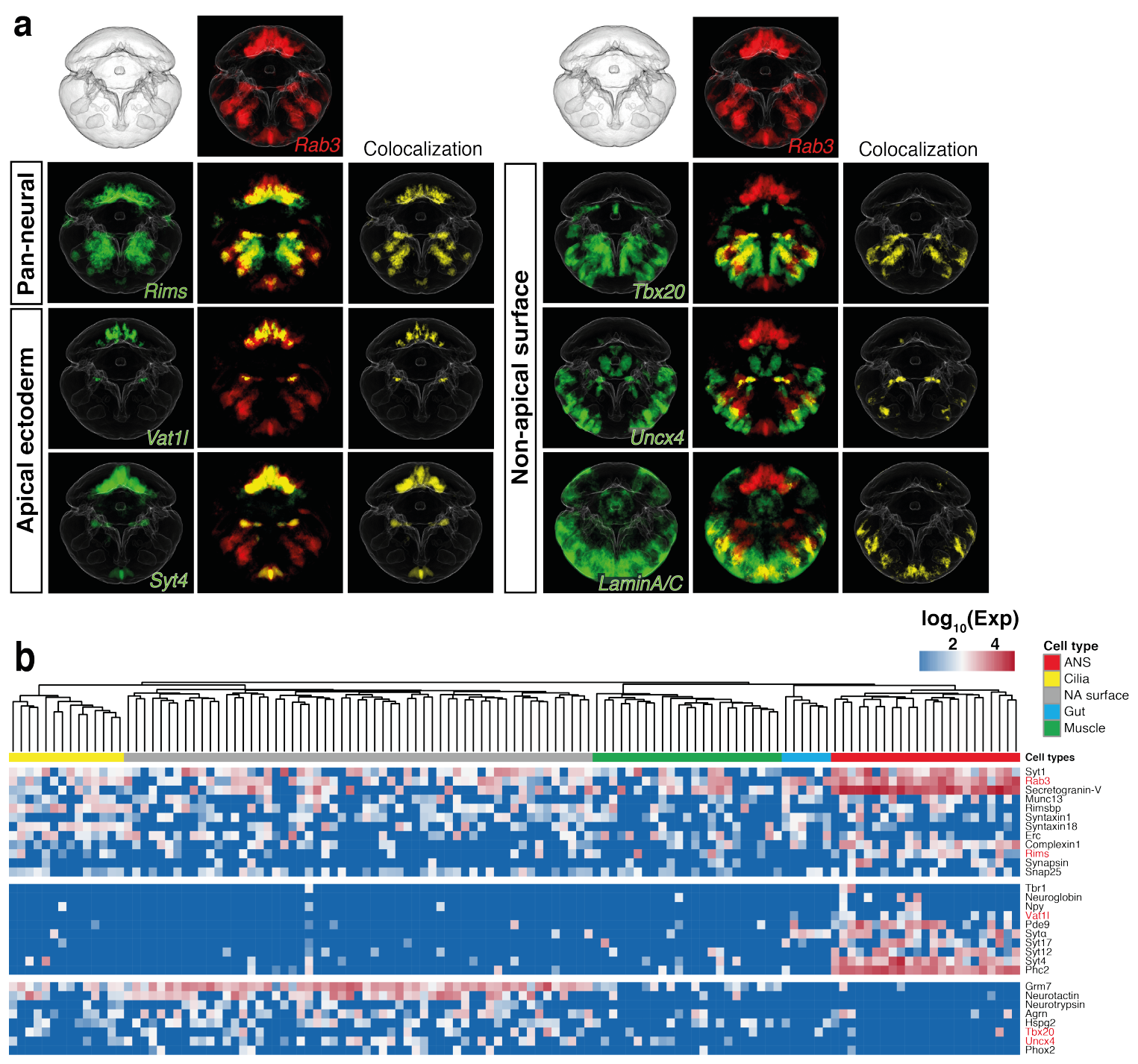

Figure 7. Shared and unique characteristics of the apical and non-apical nervous

system. a, Averaged gene expression patterns and in silico analysis of co-expression with pan-neuronal marker gene Rab3, for selected marker genes. b, Heatmap showing the expression profile of pan-neuronal markers, as well as neuronal genes specific to apical ectoderm and non-apical surface cells. Red labels, the genes for which ISH is shown in (a). 